\title{
Preparation of silver hydrosols using an acrylamide copolymer
}

\author{
Yukihito Matsuura, Mami Morimoto, Akihiro Masui and Ryoko Uda \\ Polymer Journal (2012) 44, 730-732; doi:10.1038/pj.2012.14; published online 7 March 2012
}

Keywords: acrylamide; plasmon absorption; silver nanoparticles; thermoresponsive

Recently, in the field of nanotechnology, considerable attention has been focused on the preparation of metal nanoparticles. ${ }^{1}$ In particular, a convenient method for the preparation of metal nanoparticles involving the use of organic polymers as the protecting group has been studied extensively. ${ }^{2,3}$ In general, a reducing agent is used when preparing nanoparticles protected by organic polymers. However, it has been reported that hydrosols containing gold or silver nanoparticles can be successfully prepared in a one-step process by the reduction of aqueous solutions of metal salts using poly( $N$-vinyl-2-pyrrolidone), without the use of a reducing agent. ${ }^{4}$ In this case, $\mathrm{N}$-vinyl-2-pyrrolidone is considered to act as both the reducing agent and the protecting group. These metal nanoparticles could be used for fabricating functional materials because the protecting polymers could be modified by copolymerization, with other monomers having functional groups.

Acrylamide groups have also been used as the protecting group for metal nanoparticles. ${ }^{5}$ Nanoparticles stabilized in acrylamide hydrosols have been well characterized and are known to act as functional materials. Among them, silver nanoparticles stabilized by poly ( $N$-isopropylacrylamide) (polyNIPAM) have been studied as thermoresponsive nanoparticles because polyNIPAM has a critical gelation transition at $30-40{ }^{\circ} \mathrm{C}$ owing to the formation of hydrogen bonds between the polymer chains. ${ }^{6,7}$ In these studies, a plasmon peak shift of $\sim 10 \mathrm{~nm}$ has been observed. However, in this case, the silver nanoparticles are prepared using a reducing agent, and polyNIPAM only acts as the protecting group. In this study, we prepared silver nanoparticles from a solution of $\mathrm{AgNO}_{3}$ and copolymers containing NIPAM blocks by stirring in solution, and we examined the thermoresponsive properties of the associated plasmon peaks.

\section{EXPERIMENTAL PROCEDURE}

$\mathrm{N}, \mathrm{N}$-dimethylacrylamide (DMAA), acryloylmorpholine (ACMO), and NIPAM were purchased from KOJIN Co. Ltd (Tokyo, Japan). Azobisisobutyronitrile was purchased from Wako Pure Chemical Industries Ltd (Osaka, Japan). A typical procedure for the copolymerization of acrylamides is as follows. NIPAM (1.0g), ACMO (1.0 g) and azobisisobutyronitrile (0.01g) were dissolved in $20 \mathrm{ml}$ of dioxane. After undergoing $\mathrm{N}_{2}$ gas bubbling for $20 \mathrm{~min}$, the solution was heated at $90^{\circ} \mathrm{C}$ for $12 \mathrm{~h}$. The reaction mixture was precipitated using diethylether $(300 \mathrm{ml})$ to obtain white insoluble products. The hexane solution containing the insoluble products was purified using a glass filter (pore size: $20-30 \mu \mathrm{m}$ ) and dried under vacuum overnight Figure 1 shows the chemical structures of the synthesized block copolymers. The hydrosols containing silver nanoparticles were prepared by stirring a solution of $\mathrm{AgNO}_{3}$ and the copolymer for $\sim 1-2$ weeks. Typical conditions are as follows. $\mathrm{AgNO}_{3}(100 \mathrm{mg})$ and the copolymer $(100 \mathrm{mg})$ were stirred in $10 \mathrm{ml}$ water for a week. When the color of the solution did not change sufficiently, the solution was stirred for an additional week.

Gel permeation chromatography was carried out using a Shodex GPC-101 system (SHODEX, Tokyo, Japan) in tetrahydrofuran (THF) (Wako Pure Chemical Industries Ltd.) at a flow rate of $1.0 \mathrm{ml} \mathrm{min}^{-1}$. The number-average molecular weight $(\mathrm{Mn})$ and the dispersion $(\mathrm{Mw} / \mathrm{Mn})$ were calculated on the basis of a polystyrene standard. The ratio of acrylamides in the copolymer was determined from ${ }^{1} \mathrm{H}$-nuclear magnetic resonance spectra, which were measured using a JEOL JNM-ECX400 spectrometer (JEOL Ltd, Tokyo, Japan). UV-visible spectra were measured using a Shimadzu UV-3600 spectrophotometer (SHIMADZU CORPORATION, Kyoto, Japan). The light source in the UV region (190-350 nm) was a deuterium lamp, and the source in the visible region $(330-800 \mathrm{~nm})$ was a halogen lamp. Transmission electron microscopy observations were carried out using a JEOL JEM-3100FEF microscope at a high voltage of $100 \mathrm{~V}$. The transmission electron microscopy samples were prepared by dropping the solution containing the silver nanoparticles onto a copper mesh. The fourier-transform infrared spectra of the hydrosols were measured by using the ATR method after removing the solvent by drying the hydrosols on a glass substrate.

\section{RESULTS AND DISCUSSION}

Table 1 lists the characterization data of acrylamide polymers and the results of the formation of the silver nanoparticles. The molecular weight of the homopolymer of DMAA, ACMO and NIPAM was $\sim 10^{4}$. PolyDMAA and polyACMO afforded hydrosols containing

Department of Chemical Engineering, Nara National College of Technology, Nara, Japan

Correspondence: Dr Y Matsuura, Department of Chemical Engineering, Nara National College of Technology, 22 Yatacho, Yamato-Koriyama, Nara 639-1080, Japan. E-mail: matsuura@chem.nara-k.ac.jp

Received 2 December 2011; revised 30 January 2012; accepted 5 February 2012; published online 7 March 2012 
silver nanoparticles solely by stirring the solutions, whereas the polyNIPAM solution did not afford silver nanoparticles. Because the molecular weight of these polymers was similar to each other, we inferred that the polymer block of DMAA or ACMO was suitable for preparing silver nanoparticles without using a reducing agent. Additionally, poly(DMAA-co-NIPAM) and poly(ACMO-coNIPAM) also afforded hydrosols containing silver nanoparticles. We also confirmed the preparation of the hydrosols under shading conditions.

As shown in Figures $2 \mathrm{a}$ and $\mathrm{b}$, we can confirm the formation of the silver nanoparticles. Although the numbers of the nanoparticles observed by the transmission electron microscopy measurements were not adequate, we calculated the average size of the nanoparticles and the s.d. The average size of the silver nanoparticles formed by poly(DMAA-co-NIPAM) and poly(ACMO-co-NIPAM) was $\sim 5-7 \mathrm{~nm}$. The size of nanoparticles formed by polyDMAA or polyACMO was also $5-7 \mathrm{~nm}$. As shown in Figures $3 \mathrm{a}$ and $\mathrm{b}$, the difference between the NIPAM copolymers could be observed in terms of the carbonyl peaks in the fourier-transform infrared spectra. The nanoparticles prepared from poly(DMAA-co-NIPAM) had a peak of the carbonyl group at $1600 \mathrm{~cm}^{-1}$; that is, this peak was shifted from the peak of poly(DMAA-co-NIPAM), which was at $1620 \mathrm{~cm}^{-1}$. This phenomenon was also observed in the hydrosols prepared from polyDMAA or polyACMO. This remarkable shift indicates a strong
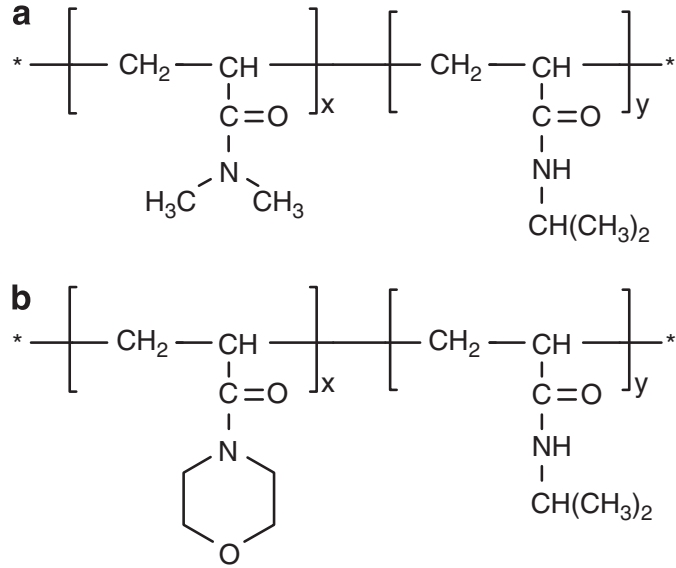

Figure 1 Polymer structure of (a) poly(DMAA-co-NIPAM) and (b) poly(ACNO-co-NIPAM). interaction between the carbonyl group and the Ag atom. In contrast, there was almost no peak shift of the carbonyl group of poly(ACMOco-NIPAM), which indicated the weak interaction between the silver atom and the carbonyl group in poly(ACMO-co-NIPAM). As mentioned in the referenced study, the reducing reagent afforded the silver nanoparticles protected by polyNIPAM. ${ }^{7}$ Therefore, ACMO blocks possibly weakened the protection of the nanoparticles.

In general, it is well known that silver nanoparticles exhibit plasmon absorption in the visible region. Hydrosols prepared from the copolymers also exhibited plasmon absorption at $\sim 400-500 \mathrm{~nm}$. After the solution changed color, the UV-visible absorption spectra remained the same for a few weeks. Figures $3 c$ and $d$ shows the temperature dependence of the UV-visible absorption spectra of the hydrosols. The hydrosols prepared from poly(DMAA-co-NIPAM) have no temperature dependence of the plasmon absorption as a result of tight protection of the nanoparticles by the DMAA blocks. Additionally, the plasmon absorption spectra of polyDMAA or polyACMO did not show temperature dependence.

On the other hand, the hydrosols prepared from poly(ACMO-coNIPAM) exhibited a broad plasmon absorption peak with thermoresponsive properties. An increase in the temperature caused a red shift of the plasmon absorption and reduced absorption, which indicated that the nanoparticle size had increased. This was possibly because the NIPAM block in the copolymers facilitated the formation of hydrogen bonding between the polymer chains when the temperature was high, and because the degree of nanoparticle protection was reduced owing to the shrinkage of the polymer blocks. In general, it is
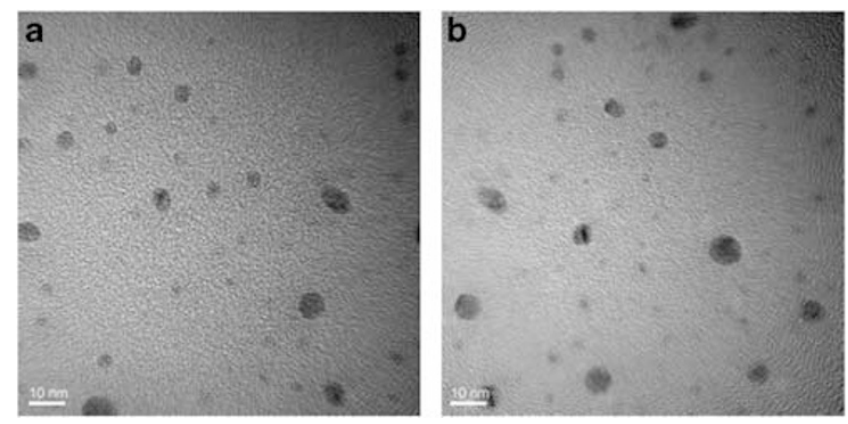

Figure 2 Transmission electron microscopy (TEM) image of silver nanoparticles prepared from (a) poly(DMAA-co-NIPAM) and (b) poly(ACNOco-NIPAM). The white bar indicates a scale of $10 \mathrm{~nm}$.

Table 1 Characteristics of polymers and nanoparticles

\begin{tabular}{|c|c|c|c|c|c|c|c|c|}
\hline \multirow[b]{2}{*}{ Polymer } & \multicolumn{2}{|c|}{$G P C$} & \multicolumn{2}{|c|}{${ }^{1} \mathrm{H}-\mathrm{NMR}$} & \multicolumn{4}{|c|}{ Nanoparticules } \\
\hline & $\mathrm{Mn} / 10^{4}$ & $M w / M n$ & $x$ & $y$ & Formation & Temperature-dependence & Average partcle size $(\mathrm{nm})$ & s.d. \\
\hline PolyDMAA & 1.29 & 1.43 & 186 & 0 & 0 & $x$ & 5.32 & 1.98 \\
\hline PolyACMO & 1.46 & 1.32 & 136 & 0 & O & $\times$ & 6.66 & 2.11 \\
\hline PolyNIPAM & 1.24 & 1.69 & 120 & 0 & $\times$ & $\times$ & - & - \\
\hline Poly(DMAA-co-NIPAM) & 1.76 & 1.50 & 124 & 116 & ○ & $\times$ & 5.85 & 2.07 \\
\hline Poly(ACMO-co-NIPAM) & 2.13 & 1.65 & 175 & 98 & O & 0 & 6.47 & 1.80 \\
\hline
\end{tabular}

Abbreviations: ACMO, acrylomorpholine; DMAA, $\mathrm{N}, \mathrm{N}$-dimethylacrylamide; GPC, gel permeation chromatography; ${ }^{1} \mathrm{H}$-NMR, ${ }^{1} \mathrm{H}$-nuclear magnetic resonance; NIPAM, n-isopropylacrylamide. 

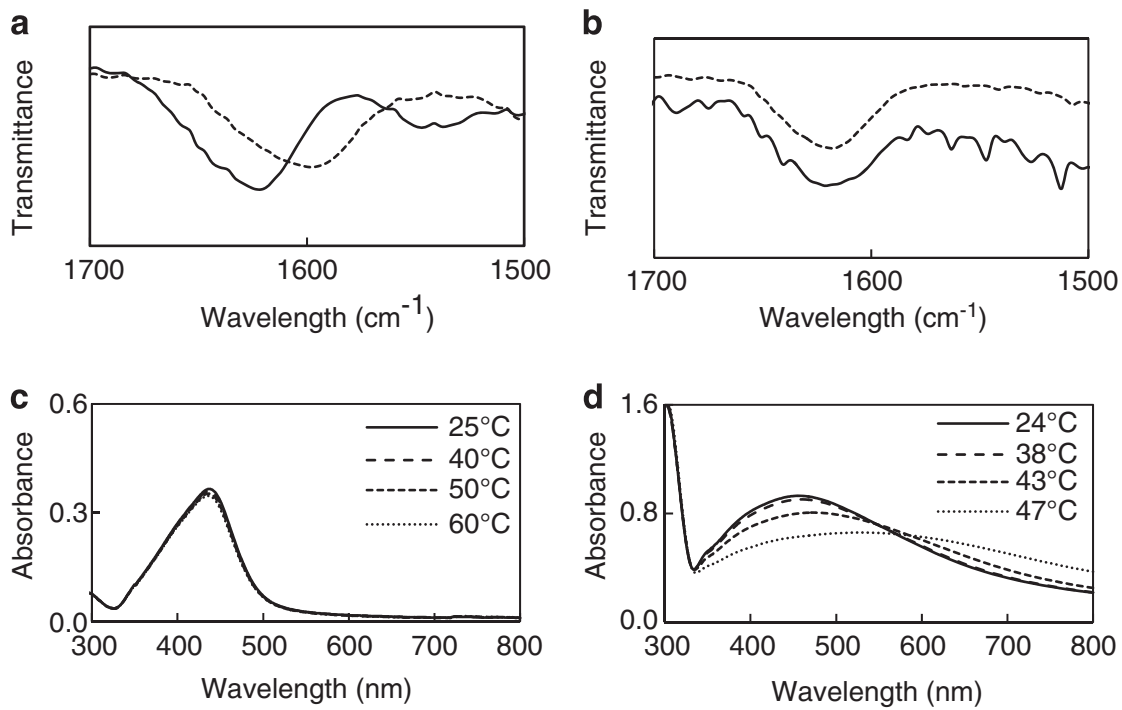

Figure 3 Fourier-transform infrared (FT-IR) spectra of (a) poly(DMAA-co-NIPAM) (solid line) and the silver nanoparticles (dotted line) and (b) poly(ACMO-coNIPAM) (solid line) and the silver nanoparticles (dotted line). UV-visible (UV-vis) absorption spectra of solutions containing silver nanoparticles prepared from (c) poly(DMAA-co-NIPAM) and (d) poly(ACMO-co-NIPAM).

well known that polyNIAPM affords a turbid solution at around $40{ }^{\circ} \mathrm{C} .{ }^{8}$ However, in our studies, turbidity of the hydrosol of poly(ACMO-co-NIPAM) was not observed at temperatures $>40^{\circ} \mathrm{C}$. The NIPAM block also has an important role in protecting the silver nanoparticles. ${ }^{7}$ Furthermore, the temperature dependence of plasmon absorption was not reversible because increasing the temperature $>60^{\circ} \mathrm{C}$ resulted in the black precipitation of silver. This result suggests that the degree of protection by poly(ACMO-co-NIPAM) is not sufficient for stabilizing the nanoparticles in the silver hydrosols, resulting in the aggregation of the nanoparticles up to $60{ }^{\circ} \mathrm{C}$. However, the thermoresponsive phenomenon could be applied to drug delivery systems by using the nanoparticles as drug carriers and for vaccine delivery.
1 Schmitd, G. (ed. Nanoparticles: from theory to application (Wiley-VCH, 2004)

2 Toshima, N. \& Yonezawa, T. Bimetallic nanoparticles-novel materials for chemical and physical applications. New J. Chem. 22, 1179-1201 (1998).

3 Naka, K., Itoh, H., Park, S. \& Chujo, Y. Synthesis of nanocomposites of metal nanopartilces utilizing miscible polymers. Polym. Bull. 52, 171-176 (2004).

4 Hoppe, C. E., Lazzari, M., Blanco, I. P. \& Quintela, M. A. L. One-Step synthesis of gold and silver hydrosols using poly(n-vinyl-2-pyrrolidone) as a reducing agent. Langmuir 22, 7027-7034 (2006)

5 Chen, M., Wang, L., Han, J., Zhang, J., Li, Z. \& Qian, D. Preparation and study of polyacryamide-stabilized silver nanoparticles through a one-pot process. J. Phys. Chem. B 110, 11224-11231 (2006).

6 Morones, J. R. \& Frey, J. R. W. Langmuir. Environmentally sensitive silver nanoparticles of controlled size synthesized with PNIPAM as a nucleating and capping agent $\mathbf{2 3}$, 8180-8186 (2007).

7 Guo, L., Nie, J., Du, B., Peng, Z., Tesche, B. \& Kleinermanns, K. Thermoresponsive polymer-stabilized silver nanoparticles. J. Colloid Int. Sci 319, 175-181 (2008).

8 Schild, H. G. Prog. Polym. Sci. 17, 163-249 (1992). 